\title{
Wind-induced Spatial and Temporal Variations in the Thermohaline Front in the Jeju Strait, Korea
}

\author{
In-Seong Han ${ }^{1}$, Young-Sang Suh ${ }^{1 *}$ and Ki-Tack Seong ${ }^{2}$ \\ ${ }^{1}$ Fishery and Ocean Information Division, National Fisheries Research and Development Institute, Busan 619-902 Korea \\ ${ }^{2}$ Southwest Sea Fisheries Research Institute, National Fisheries Research and Development Institute, Yeosu 556-823 Korea
}

\begin{abstract}
We investigated the short-term and local changes in the thermohaline front in the Jeju Strait, Korea, which is usually formed during winter and spring. To do so, we compared Real-Time Observation System by Ferryboat (RTOSF) data with wind data and routinely collected oceanographic data. During February and April 2007, a thermohaline front formed in the Jeju Strait around the $13-14^{\circ} \mathrm{C}$ isotherms and 33.0-33.5 isohalines. The thermohaline was clearly weakened and began moving southward in mid-March. The variations in the surface temperature and salinity showed a continuous north-south oscillation of the thermohaline front with a period of 3-10 days. The speed of the short-term and local fluctuation of thermohaline front was about 5-30 cm/s. We confirmed these findings by examining the variation in the maximum temperature gradient and $14^{\circ} \mathrm{C}$ isotherm during the study period. These short-term and local changes had not been previously detected using serial oceanographic and satellite data. Analysis of local wind data revealed a northerly wind fluctuation with a period of 3-10 days, which was clearly related to the short-term and local changes in the thermohaline front. The short-term and local changes of the thermohaline front in the Jeju Strait originated from local changes in the winter monsoon in this area.
\end{abstract}

Key words : Thermohaline Front, Ferryboat monitoring, Jeju Strait, Wind-induced condition, Cheju Current, Korean Coastal Water

\section{Introduction}

The Jeju Strait located between Jeju Island and the southern coast of Korea, is a relatively wide strait of about $70 \mathrm{~km}$ in both length and width. The mean water depth in the strait is about $100 \mathrm{~m}$ and maximum water depth is about $120 \mathrm{~m}$ in the southern part of the strait. The strait has become an important research site for studies on circulation in the East China Sea, the Yellow Sea, and near the Korea/Tsushima Strait. Other important research topics in the strait include the recent outbreaks of harmful algal blooms, the transport of large jellyfish, the approach of a low salinity water mass, and the appearance of a low temperature water mass.

A mean eastward current, which is usually called the Cheju Current or Jeju Current, has been documented in the Jeju
Strait by in-situ observation results (Chang et al., 1995; Pang et al., 2003). Chang et al. (2000) showed that the Cheju Current mostly carries water of high temperature and salinity from the Kuroshio Current in winter and spring. They also reported that the strong core of the eastward component of the Cheju Current is found near Jeju Island with a maximum speed about $17.0 \mathrm{~cm} / \mathrm{s}$ and volume transport of 0.37 to $0.45 \mathrm{~Sv}$ in the winter and spring. Water masses in the Jeju Strait are usually composed of warm water originating from the Tsushima Warm Current, Yellow Sea Cold Water, and Korean Southern Sea Cold Water (Son et al., 2003).

The thermohaline front in the Jeju Strait was first reported by Gong (1971) in a study of the oceanic environment of fish-
Open Access http://dx.doi.org/10.5657/FAS.2013.0117

This is an Open Access article distributed under the terms of the Creative Commons Attribution Non-Commercial License (http://creativecommons. org/licenses/by-nc/3.0/) which permits unrestricted non-commercial use, distribution, and reproduction in any medium, provided the original work is properly cited. pISSN: 2234-1749 eISSN: 2234-1757
Received 6 March 2013; Revised 27 March 2013

Accepted 27 March 2013

*Corresponding Author

E-mail: yssuhkorea@korea.kr 
ing grounds in the area. He noted that the sharpest and most stable front between the Tsushima Warm Current and the South Korean Coastal Water occurred in autumn and winter near the Jeju Strait. In winter, a tongue-shaped thermohaline front with a horizontal form similar to the Greek letter ' $\Gamma$ ' was established at the southern entrance of the Yellow Sea and west of Jeju Island (Lee, 1983; Lie, 1985; Chen et al., 1994; Lie et al., 2000; Son et al., 2003). This ' $\Gamma$ ' shape varied frequently according to the behaviors of the Yellow Sea Cold Water and the Tsushima Warm Current, which are considered to be the origin of the Yellow Sea Warm Current (Lie et al., 2001). Seung and Shin (1996) used a simple numerical model to show that this thermohaline front is formed by the wind-driven advection of local cooled water to the southern warm area. The thermohaline front area in the Jeju Strait has also long been known as a good fishing ground because of its high concentration of nutrients and high primary productivity (Gong, 1971; Kang and Jeon, 1999; Yang et al., 1999; Kim et al., 2005). Thus, research regarding the frontal area is important to fisheries research.

The studies cited above have provided important information regarding the formation and structure of the thermohaline front near the Jeju Strait. However, those studies could not describe short-term and local changes in the thermohaline front because of data limitations. Specifically, the bimonthly observation interval of serial oceanographic investigations in Korean waters cannot reveal short-term changes, and continuous monitoring by satellite hindered by the frequently cloudy weather conditions.

In situ observations from research vessels have also become more difficult because of rising fuel prices and ship scheduling. However, commercial vessels are increasingly participating in programs to aid marine environmental research, such as the Ship-of-Opportunity Program (SOOP). Measurements taken from ferryboats and merchant ships can provide oceanographic data along various routes and frequencies. To clarify the complex oceanic conditions, various types of oceanographic data must be accumulated. For these reasons, Korea's National Fisheries Research and Development Institute (NFRDI) has operated a program since 2006 by which ferryboats provide real-time observations across the Jeju Strait.

This study examined short-term and local changes in the thermohaline front across the Jeju Strait. Data collected by the real-time ferryboat observation system from February to April 2007 were compared with in-situ observations by research vessels and to satellite remote sensing results. We also examined the causes of short-term and local changes in the thermohaline front such as variations in the wind direction and speed. Understanding the short-term and local changes in the thermohaline front is important clarifying the changes in fishing ground conditions and oceanic ecosystems in the area.

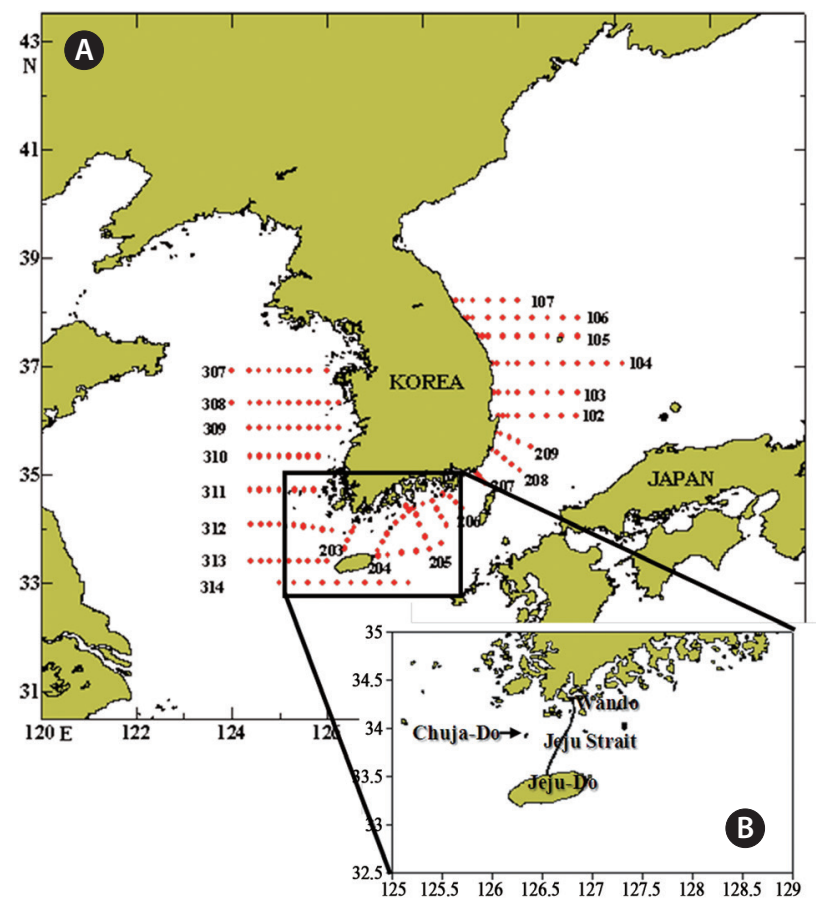

Fig. 1. Study map (A) and the location of Real-Time Observation System by Ferryboat (RTOSF) operation across the Jeju Strait (B).

\section{Materials and Methods}

The real-time observation system by ferryboat (RTOSF) program across the Jeju Strait began on 28 August 2006, using the vessel Hanil Car ferry-2 (3,211 G/T, maximum speed about 18 knots; Hanil Express Co. Ltd., Seoul, Korea). This ferryboat makes a daily roundtrip between Jeju and Wando across the Jeju Strait (Fig. 1). We installed a SBE-45 ThermoSalino-Graph (Sea-Bird Electronics, Inc., St. Bellevue, WA, USA) in the engine room with a differential global positioning system (DGPS) and code division multiple access (CDMA) antenna on the deck of the ferryboat. This system was first used in late August 2006 and has been operating stably since mid-January 2007. The system measures the surface temperature and salinity at 10-min intervals when the ship is moving faster than 3 knots. Temperature, salinity, and location data are then transmitted to the NFRDI server via CDMA communication in real-time. In this study, we used the daily roundtrip temperature and salinity data.

NFRDI has been conducting bimonthly serial oceanographic investigations at 196 stations in Korean waters since 1961. Measurements include temperature, salinity, dissolved oxygen, zooplankton, phytoplankton and nutrients. In this study, we examined temperature and salinity data collected as serial oceanographic investigations data by NFRDI in February and April 2007 around the South Sea of Korea.

We also examined weekly composite images of sea surface temperature (SST) data observed by the National Oceanic and 

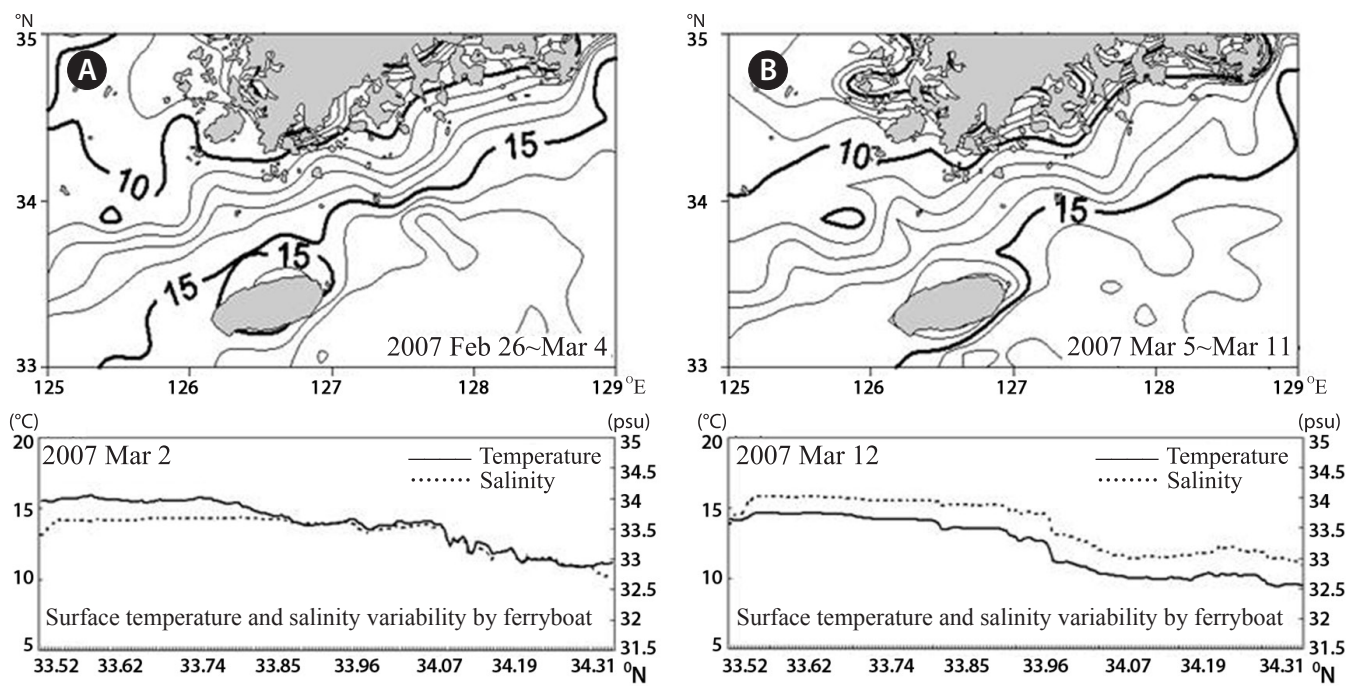

Fig. 2. Satellite weekly composite images (upper panels) and the variation of surface temperature (sold line) and salinity (dashed line) by Real-Time Observation System by Ferryboat (RTOSF) (lower panels) on early March (A) and mid-March (B).

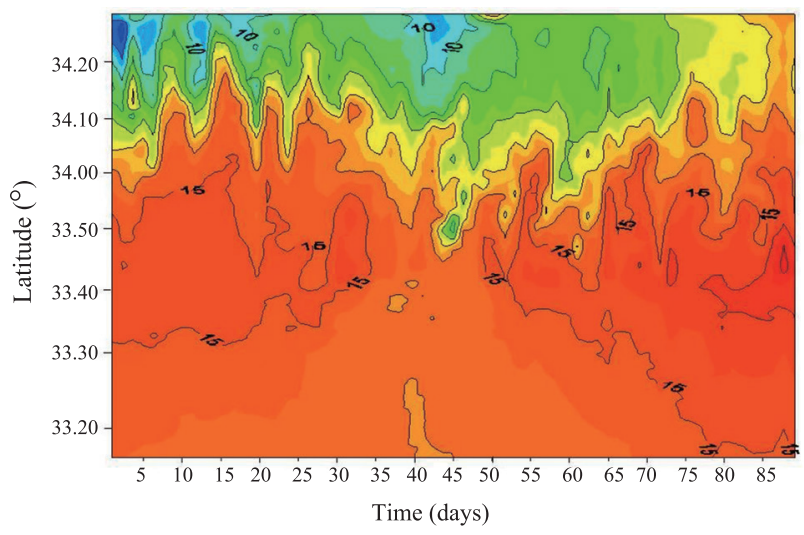

Fig. 3. Temporal and spatial variation of sea surface temperature by Real-Time Observation System by Ferryboat (RTOSF) during February and April 2007. X-axis indicates days from February 1, 2007 and Y-axis indicates latitude in the Jeju Strait, respectively.

Atmospheric Administration (NOAA)/Advanced Very High Resolution Radiometer (AVHRR) around the South Sea of Korea. These images were obtained from the Satellite Ocean Information Laboratory of NFRDI.

Wind direction/speed data at Chuja Island, located near the entrance of the Jeju Strait, in February, March, and April 2007 provided the Korea Meteorological Agency (KMA) were also examined in relation to the short-term and local thermohaline changes across the Jeju Strait.

Using these data sets, we examined the short-term and local changes in the thermohaline across the Jeju Strait. The thermal front in this area was previously defined as having a spatial temperature gradient larger than $0.186^{\circ} \mathrm{C} / \mathrm{mile}$ (Yang et al., 1998, 1999). As the definition of a thermal front or thermohaline front is relative, here we define the thermohaline as having a spatial temperature gradient larger than $0.1^{\circ} \mathrm{C} / 10^{3} \mathrm{~m}$.

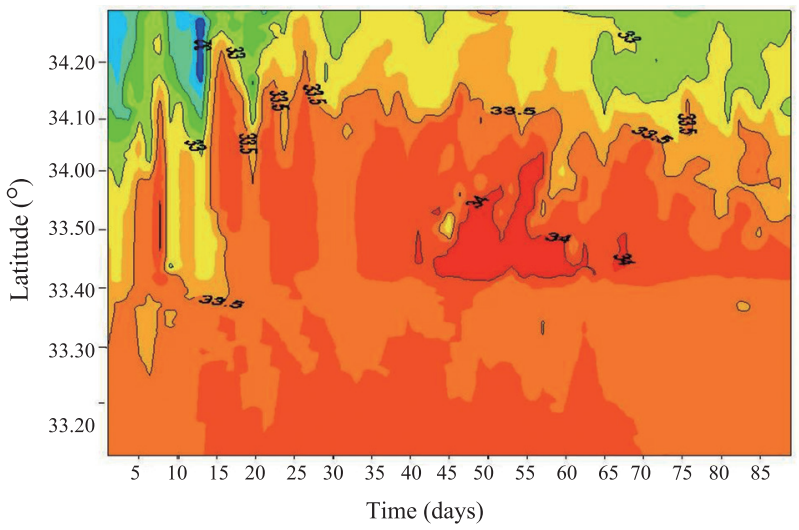

Fig. 4. Temporal and spatial variation of sea surface salinity by RealTime Observation System by Ferryboat (RTOSF) during February and April 2007. X-axis and Y-axis indicate same as Fig. 3.

\section{Results}

\section{Surface temperature and salinity variations across the Jeju Strait}

We examined the spatial temperature distribution shown by the satellite images and the RTOSF data across the Jeju Strait. Fig. 2A and B shows the NOAA/AVHRR SST weekly composite images of the southwestern area from 26 February to 4 March and from 5 March to 11 March 2007. The figure also shows the spatial variations in the SST measured by RTOSF on 2 and 12 March 2007 across the Jeju Strait. Considering the ferryboat route, the spatial variations in SST obtained by RTOSF closely corresponded to the temperature distribution shown by the satellite composite images. Furthermore, the temporal variations in sea surface salinity (SSS) were also 

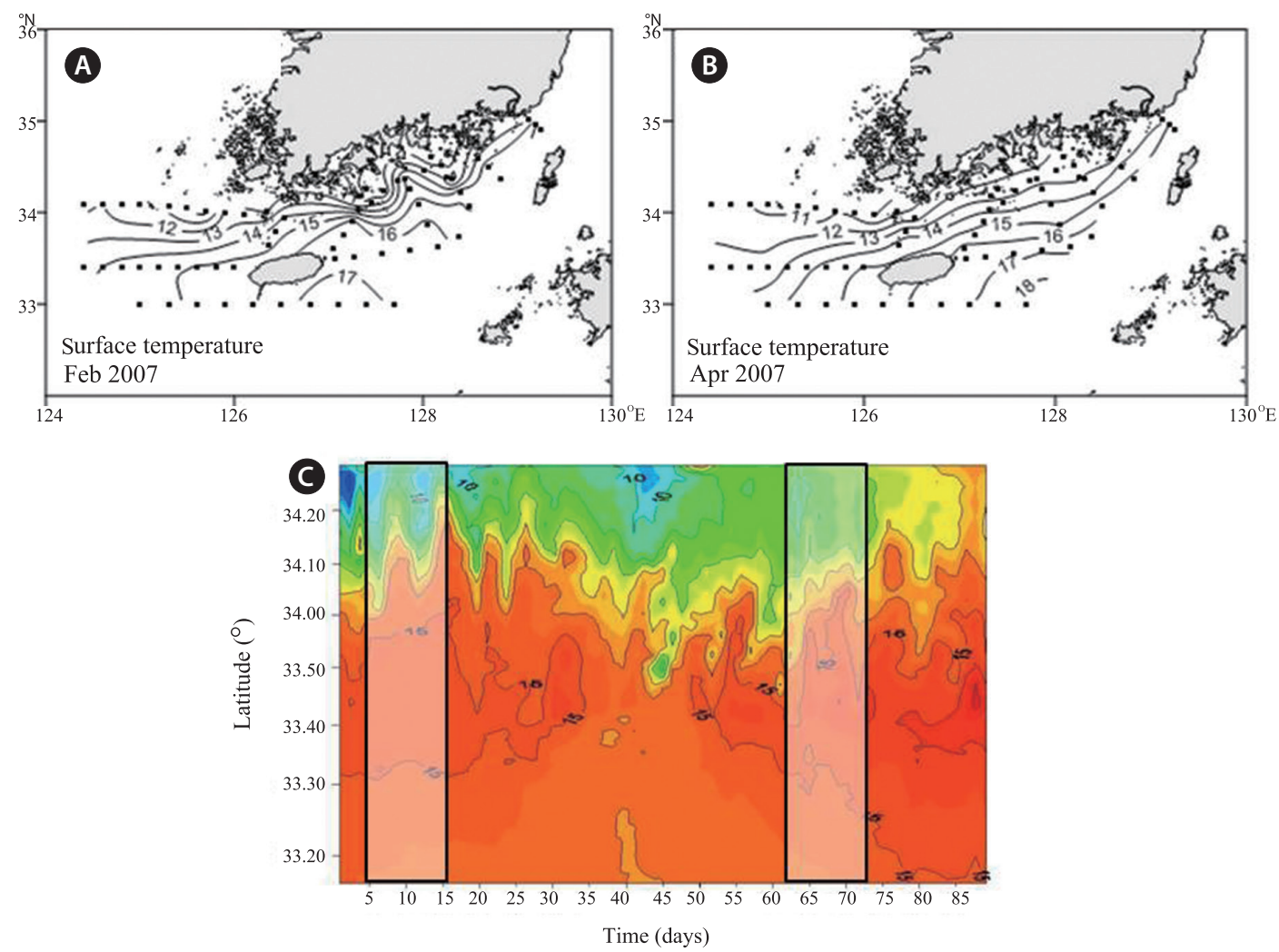

Fig. 5. The distributions of sea surface temperature by National Fisheries Research and Development Institute (NFRDI)'s serial oceanography investigations of in February (A) and April (B) 2007 with temporal and spatial variation of sea surface temperature by Real-Time Observation System by Ferryboat (RTOSF) (C), same as Fig. 3. Shaded areas indicate the periods of NFRDI conductivity-temperature-depth (CTD) casts.

very similar to the temporal variations in SST measured by RTOSF. These values suggest that the thermohaline front was formed around $34^{\circ} \mathrm{N}$ in the Jeju Strait in early or mid-March 2007.

In the spatial and temporal variations in the RTOSF SST from 1 February to 30 April 2007 (Fig. 3), a strong thermal front, with a spatial temperature gradient larger than $0.2^{\circ} \mathrm{C}$ $/ 10^{3} \mathrm{~m}$, was clearly formed at $34.0-34.1^{\circ} \mathrm{N}$ in early February and rema ined until late February. Subsequently, the thermal front moved southward and was slightly weakened after early March. The front was markedly weakened and could not be clearly determined after early April.

In the spatial and temporal variations in the SSS from 1 February to 30 April 2007 (Fig. 4), a strong and distinct salinity front was identified around $33.4-34.1^{\circ} \mathrm{N}$ during February, but gradually weakened after mid-March.

The thermohaline front with north-south oscillation of 3-10 days between the South Korean Coastal Water and the Tsushima Warm Current are also shown in Figs. 3 and 4. The South Korean Coastal Water has low temperature and salinity and originates from high latitudes of the Yellow Sea; the Tsushima Warm Current has high temperature and salinity and originates from low latitudes (Son et al., 2003). The thermohaline front usually formed around $13-14^{\circ} \mathrm{C}$ isotherms and $33.0-33.5$ isohalines during this period.

\section{Comparison of ferryboat observations and satel- lite investigations}

During the study period, NFRDI conducted two serial oceanographic investigations around the South Sea of Korea in early February and early April. We compared the RTOSF surface temperature and salinity variations with those shown by the NFRDI conductivity-temperature-depth (CTD) casts in February and April. Fig. 5A and B shows the distributions of surface temperature in early February and April, and Fig. $5 \mathrm{C}$ presents the RTOSF temporal and spatial variations in (the same as Fig. 3). The shaded areas in Fig. 5C indicate the periods of the NFRDI CTD casts. In these figures, we could not isolate a significant thermal front, especially in early February. The thermal front in early April was stronger than that in early February. However, in the RTOSF data, the thermal front was distinctively stronger in early February than in early April. The serial oceanographic investigations by CTD casts were conducted at only a small number of stations, which likely explains the difference in the results. During strong thermal 

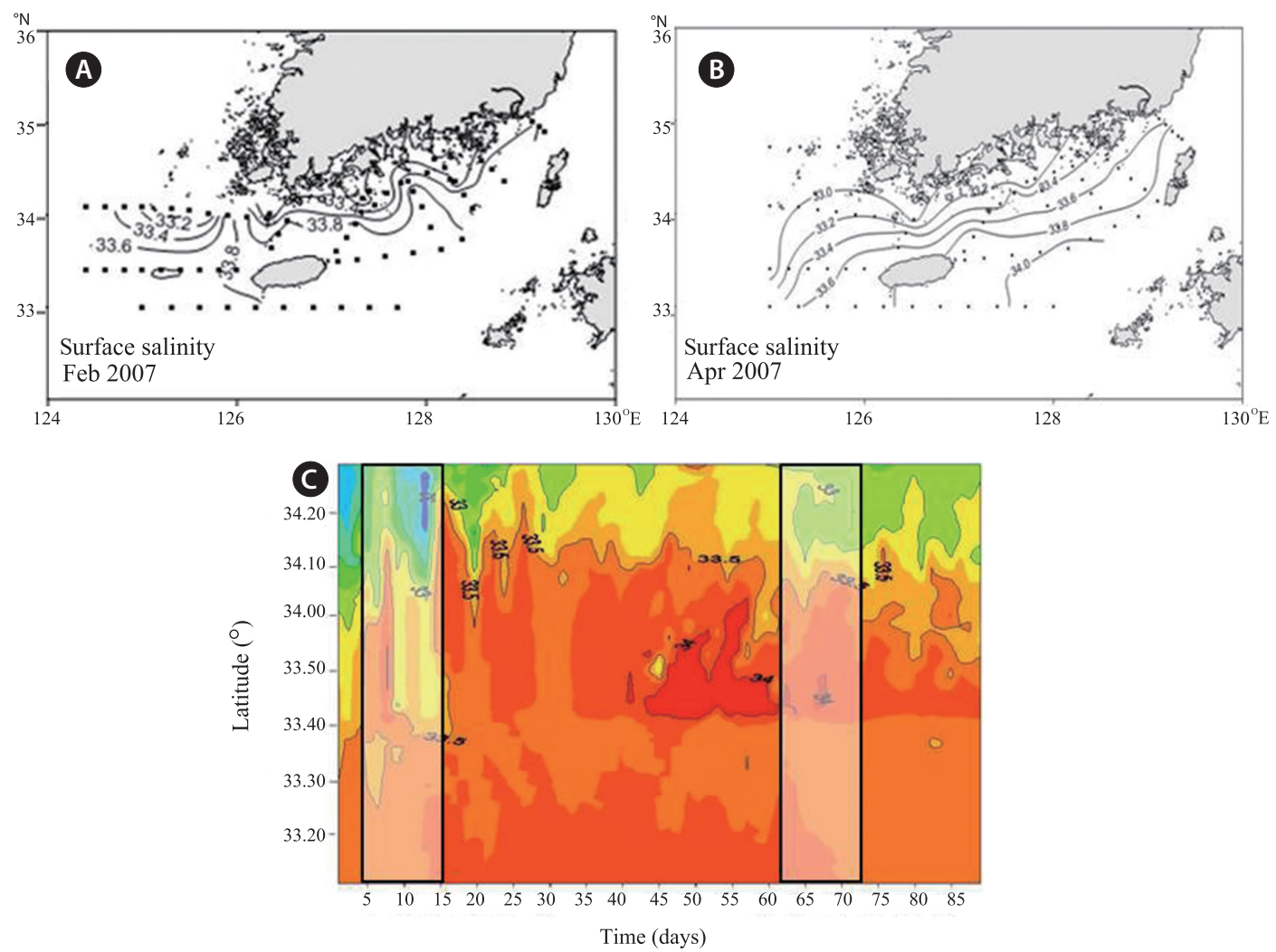

Fig. 6. The distributions of sea surface salinity by National Fisheries Research and Development Institute (NFRDI)'s serial oceanography investigations in February (A) and April (B) 2007 with temporal and spatial variation of sea surface salinity by Real-Time Observation System by Ferryboat (RTOSF) (C), same as Fig. 4. Shaded areas indicate the periods of NFRDI conductivity-temperature-depth (CTD) casts.

fronts, such as that formed around the coastal area in early February, the limited CTD observations were not sufficient to show the real oceanography phenomena. Therefore, we cannot discern the detailed variation in the thermohaline front in this area during winter and spring from historical serial oceanography investigations, although these investigations have been conducted continuously for over 40 years.

Fig. 6 shows the surface salinity distributions from serial oceanography investigations with the RTOSF temporal and spatial variations in salinity, similar to Fig. 5 . We detected the same phenomena in the case of surface salinity, as found for surface temperature. The salinity front could not be clearly determined in the serial oceanography investigation across the Jeju Strait because of the limited station numbers, especially in the coastal area.

In Fig. 7, we further examine the temperature distributions by comparing the RTOSF data to weekly NOAA/AVHRR composite images of surface temperature for the period of early February to late April 2007 around the Jeju Strait. The figure does not show the complex isotherms around Jeju Island and near the south coast of Korea. From the satellite images, we determined that the thermal front gradually weakened and moved southward from winter to spring, similar to the
RTOSF result. However, these satellite images do not show the short-time variations in the thermal front, namely the 3-10 days north-south oscillation of the thermohaline front. This is because poor weather conditions limited the amount of usable satellite images. Thus, the RTOSF observations provided more detailed information on the variation of the thermohaline front in this area.

\section{Wind-induced short-term changes in the thermo- haline front across the Jeju Strait}

Above, we determined that the thermohaline front gradually weakened from winter to spring. To examine the shortterm changes in the thermohaline front using the RTOSF data, we show the temporal variations in the maximum temperature gradient and maximum temperature gradient location (by latitude) in Fig. 8A and B. Data for maximum temperature gradients smaller than $0.2 \times 10^{-3 \circ} \mathrm{C} / \mathrm{m}$ were not included in the figure for clarify. These two time series also showed the north-south oscillation of the thermohaline front with a period of about 3-10 days, although detailed variations were not shown. The temperature gradient in the thermohaline front was clearly weakened and the thermohaline front slowly moved south- 

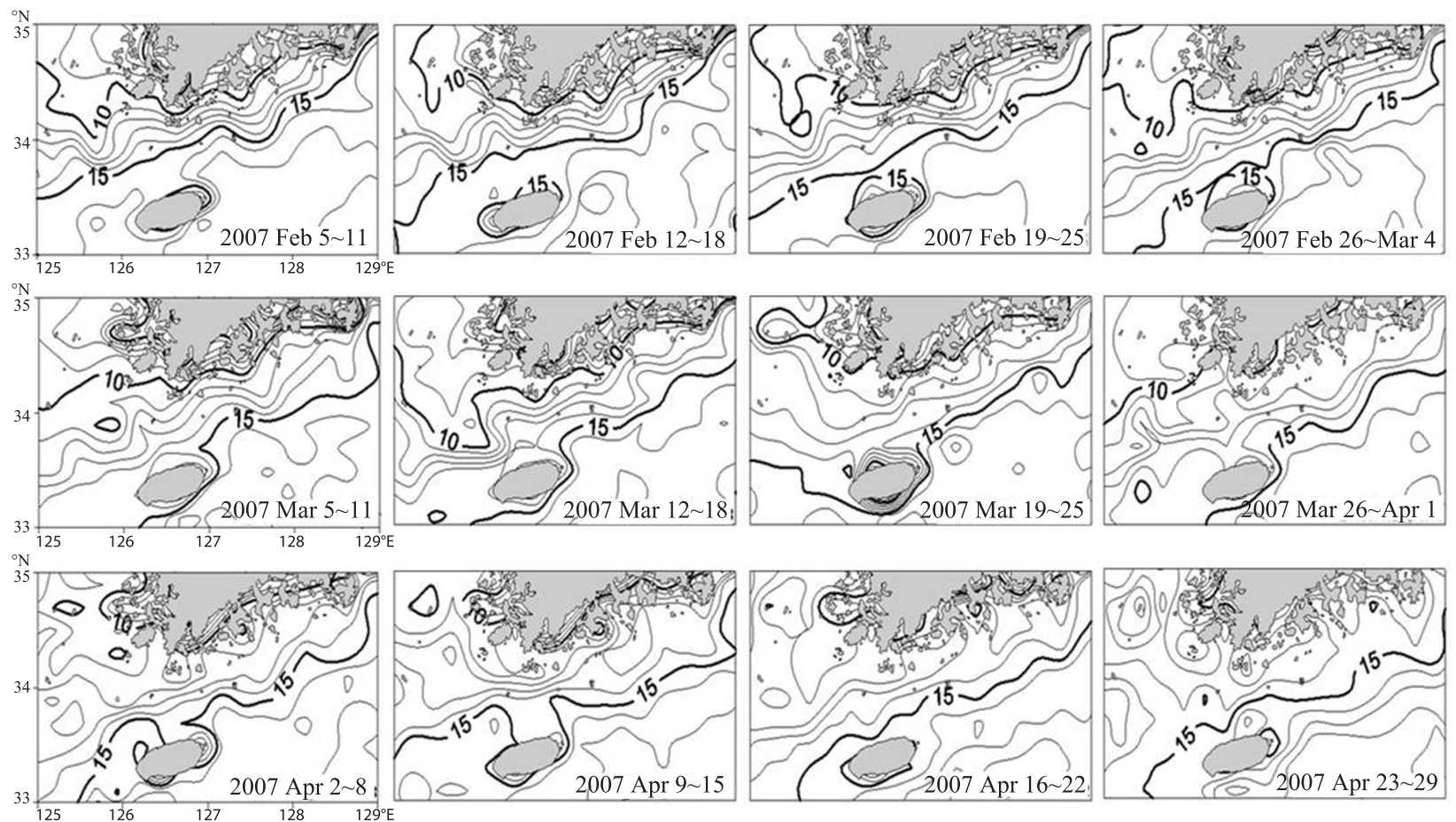

Fig. 7. Weekly composite images of sea surface temperature by National Oceanic and Atmospheric Administration (NOAA)/Advanced Very High Resolution Radiometer (AVHRR) from early February to late April 2007. The characters in these figures indicate the composite period.
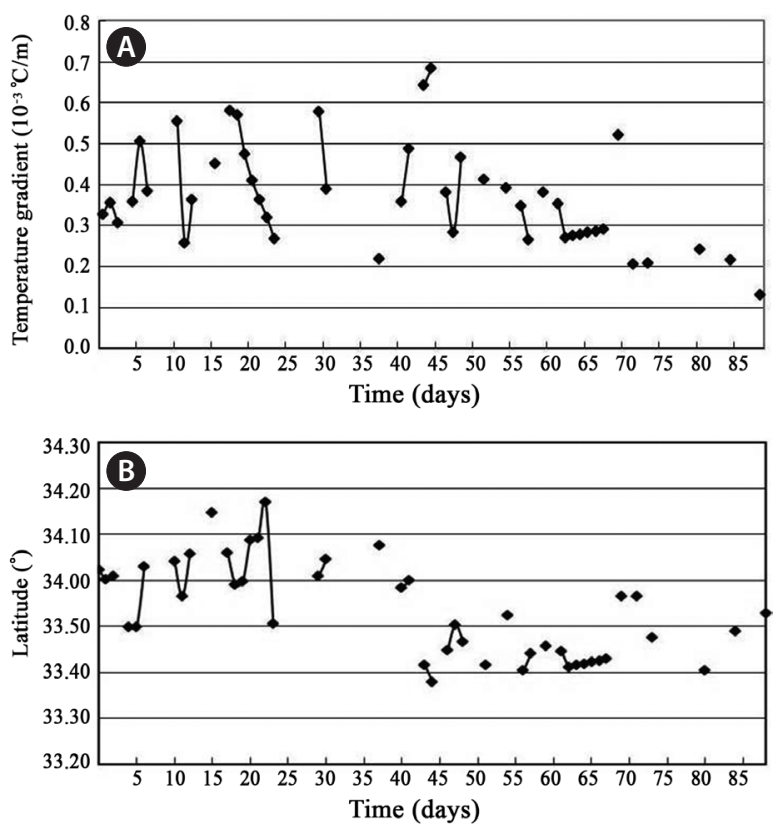

Fig. 8. Time series of the temporal variation of maximum temperature gradient (A) and of maximum temperature gradient location (B) in the Jeju Strait by Real-Time Observation System by Ferryboat (RTOSF) data. If the maximum temperature gradient were smaller than $0.2 \times 10^{-30} \mathrm{C} / \mathrm{m}$, the data were not marked to prevent confusing the thermohaline conditions in two time series. X-axis indicates days from 1 February 2007. ward from mid-March. Despite an insufficient amount of data, the results indicated when the maximum temperature gradient was located in a coastal area (i.e., the thermohaline front was located in the northern part of the Jeju Strait), the temperature gradient of the thermohaline front seemed to be strong. On the other hand, when the temperature gradient of the thermohaline front seemed to weaken, the thermohaline front moved southward.

During our study period, the thermohaline front usually formed around the $14^{\circ} \mathrm{C}$ isotherm across the Jeju Strait. We examined the temporal variation in the $14^{\circ} \mathrm{C}$ isotherm location in the Jeju Strait during the study period, as shown in Fig. 9A. This time series also clearly shows the north-south oscillation of the thermohaline front. Furthermore, the $14^{\circ} \mathrm{C}$ isothermal variations also indicate that the thermohaline front had a period of about 3-10 days and moved southward from mid-March. We could also compute the speed of the northsouth oscillation of the thermohaline front by this time series, which was estimated to be about $5-30 \mathrm{~cm} / \mathrm{s}$.

To examine the mechanism of this short-term variation in the thermohaline front, we compared it with surface wind variations, determined by hourly wind data measured at Chuja Island. Fig. 9B and $\mathrm{C}$ shows the wind direction and wind speed at Chuja Island from February to April 2007. During this period, northwesterly wind dominated around the Jeju 

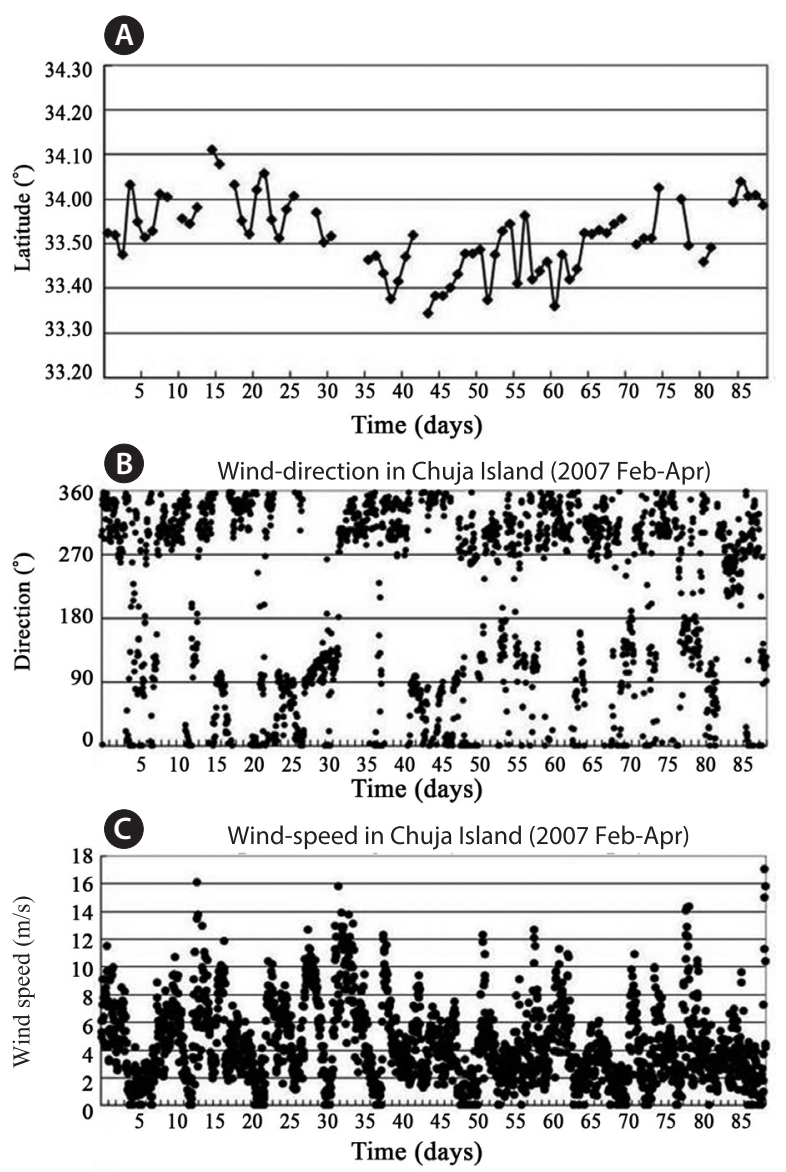

Fig. 9. Temporal variation of $14^{\circ} \mathrm{C}$ isotherm location (A) during the study period by Real-Time Observation System by Ferryboat (RTOSF) data, wind direction (B) and wind speed (C) in Chuja Island during February and April 2007.

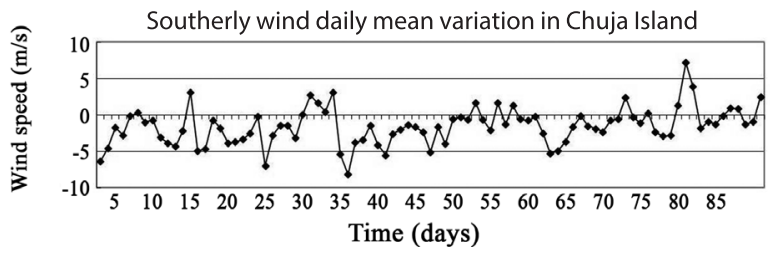

Fig. 10. Temporal variation of daily mean southerly wind speed at Chuja Island during February and April 2007.

Strait. However, the wind direction sometimes changed to southerly with a period of about a few days. The wind speed of the northerly wind was significantly stronger than that of the southerly wind. Fig. 10 shows the time series of southerly wind daily mean variation compared with the thermohaline front variation (from Fig. 3) during the study period. The daily mean southerly wind at Chuja Island showed short-term variation with a period of about a few days, similar to the shortterm change in the thermohaline front. This suggests that the similar to the north-south oscillation of the Jeju Strait thermohaline front with a period of is closely related to short-term changes in the winter monsoon and the strength of the northerly wind.

Chang and Kim (1995) reported that low-frequency current fluctuations with a period of about a few days predominated in the along-strait current component based on 20 days of data from a mooring experiment in Jeju Strait during mid-April and early May. Their examination of current and wind variations suggested that the along-strait current fluctuations were poorly related to the local wind field at Jeju Island. However, in the present study, short-term spatial and temporal changes in the thermohaline front were found to relate to local changes in the wind field during late winter and spring.

\section{Discussion}

The thermohaline front in the Jeju Strait usually forms in winter and early spring between the Korean coastal Water and the Tsushima Warm Current. However, short-term and local changes in the thermohaline front had been unknown because of the limited number of observation stations and periods. Short-term variation in the thermohaline front is very important to fisheries activities and oceanography phenomena around the study area. NFRDI has operated a ferryboat-based real-time observation system (RTOSF) in the Jeju Strait since late August 2006. This system provides continuous, high-frequency surface temperature and salinity data. Using RTOSF data collected in late winter and early spring 2007, we examined the short-term and local changes in the thermohaline front in the Jeju Strait. We then compared the RTOSF results with the results obtained from hydrographic investigations and satellite observations.

The 3 months of RTOSF observations examined in this study showed that the north-south oscillation of the thermohaline front in the Jeju Strait fluctuated with the period of about 3-10 days. The thermohaline front markedly weakened and moved to southward from mid-March. The estimated speed of the north-south oscillation of the thermohaline front in the Jeju Strait during the study period was about $5-30 \mathrm{~cm} / \mathrm{s}$. This north-south oscillation of the thermohaline front could not be determined from the satellite images of surface temperature or from serial oceanography investigations, even though such investigations have collected large amounts of observational data. This is likely because of the limited time intervals of sampling and the small number of observation stations. We also examined variations in the maximum temperature gradient and $14^{\circ} \mathrm{C}$ isotherm during this period to identify short-term and local change in the thermohaline front. These results also showed that the thermohaline front in the Jeju Strait fluctuated with a period about 5-30 days and weakened and moved southward from mid-March. Furthermore, analysis of wind data measured at Chuja Island showed that the short-term and 
local changes in the thermohaline front clearly related to the short-term changes in the winter monsoon in this area. The southerly wind speed also had a period of about 3-10 days.

This study examined the local changes in the thermohaline front in the Jeju Strait during a period of a few months. The thermohaline front usually formed from northwest of Jeju to the Korea/Tsushima Strait with a length of about $200 \mathrm{~km}$. Understanding of mechanisms that create fluctuations in the thermohaline front in the South Sea of Korea during winter and spring is important to understanding the fisheries condition in the area. In the future, we plan to examine fluctuations across the entire thermohaline front using data from other RTOSF ferryboat that travel the Busan-Jeju route and Busan-Hakata route.

\section{Acknowledgements}

We would like to thank to captain and crews of Hanil Car ferry-2 (Hanil Express Co. Ltd.) for providing the chance of RTOSF observation. This study was funded by a grant from National Fisheries Research and Development (RP-2013-ME004 ).

\section{References}

Chang KI and Kim K. 1995. Sub-tidal flow in the Cheju Strait. Yellow Sea 1, 93-103.

Chang KI, Kim K, Lee SW and Shim TB. 1995. Hydrography and subtidal current in the Cheju Strait in Spring, 1983. J Korean Soc Oceanogr 30, 203-215.

Chang KI, Suk MS, Pang IC and Teague WJ. 2000. Observations of the Cheju Current. J Korean Soc Oceanogr 35, 129-152.

Chen C, Beardsley RC, Limeburner R and Kim K. 1994. Comparison of winter and summer hydrographic observations in the Yellow Sea and East China Sea and adjacent Kuroshio during 1986. Cont Shelf
Res 14, 909-929.

Gong Y. 1971. A study on the South Korean Coastal front. J Oceanogr Soc Korea 6, 25-36.

Kang YS. and Jeon KA. 1999. Biological and chemical characteristics and trophodynamics in the frontal zone in the southern waters of Korea. J Korean Fish Soc 32, 22-29.

Kim JI, Kim JY, Choi YK, Oh HJ and Chu EK. 2005. Distribution of the anchovy eggs associated with coastal frontal structure in southern coastal waters of Korea. Korean J Ichthyol 17, 205-216 (in Korean with English abstract).

Lee JC. 1983. Characteristics of front near the Cheju Strait in early winter. Bull Korean Fish Soc 16, 51-58.

Lie HJ. 1985. Wintertime temperature-salinity characteristics in the southeastern Hwanghea (Yellow Sea). J Oceanogr Soc Jpn 41, 291-298.

Lie HJ, Cho CH, Lee JH, Lee S and Tang Y. 2000. Seasonal variation of the Cheju Warm Current in the Northern East China Sea. J Oceanogr 56, 197-211.

Lie HJ, Cho CH, Lee JH, Lee S, Tang Y and Zou E. 2001. Does the Yellow Sea Warm Current really exist as a persistent mean flow? J Geophys Res 106, 22199-22210.

Pang IC, Hong CS, Chang KI, Lee JC and Kim JT. 2003. Monthly variation of water mass distribution and curent in the Cheju Strait. J Korean Soc Oceanogr 38, 87-100.

Seung YH and Shin SI. 1996. A simple model of the formation of thermo-haline front in the southeastern Yellow Sea in winter. J Korean Soc Oceanogr 31, 23-31.

Son YT, Lee SH, Lee JC and Kim JC. 2003. Water masses and frontal structures in winter in the Northern East China Sea. The Sea 8, 327-339 (in Korean with English abstract).

Yang YJ. Kim SH and Rho HK. 1998. A study on the temperature fronts observed in the South-West Sea of Korea and the Northern area of the East China Sea. J Korea Fish Soc 31, 695-706.

Yang YJ, Kim SH, Rho HK and Jeong DG. 1999. Relationship between SST fronts and pulse-seine fishing grounds in the South-West Sea of Korea and the Northern area of the East China Sea. J Korea Fish Soc 32, 618-623. 